\title{
Design for Reliability Highly Responsible Systems on the Example of a Moving Rod
}

\author{
Yury P. Pokhabov* \\ JSC "NPO PM SDB" \\ 55a Lenin Str., Zheleznogorsk, 662972, Russia
}

Received 06.08.2019, received in revised form 02.09.2019, accepted 17.09.2019

\begin{abstract}
The article is devoted to one of the design technologies of highly responsible systems on the example of a rotating rod of a spacecraft. This technology allows take into account the specified requirements for reliability from the very first design solutions. An algorithm for constructing a generalized parametric model of the functioning of products is proposed, which is intended for carrying out the calculated and experimental verification of the output parameters of functioning and their correlation with the given reliability indicators. The methodology of the design \& technological reliability analysis is presented, which allows performing analytical verification of the functioning parameters in order to identify necessary and sufficient requirements for performing computational and experimental verification and manufacturing products with a given reliability. This design technology, based on parameterization and design \& technological reliability analysis, makes it possible to achieve reliability of mechanical devices whp $R \equiv 1$, regardless of the type of mass production. At the same time, potential failures can be successfully eliminated or mitigated at the earliest stages of the life cycle (design and engineering), and the possible risks of the occurrence of failures are promptly evaluated.
\end{abstract}

Keywords: design, design for reliability (DFR), highly responsible systems, spacecraft, design \& technology reliability analysis (DTRA).

Citation: Pokhabov Yu.P. Design for reliability highly responsible systems on the example of a moving rod, J. Sib. Fed. Univ. Eng. technol., 2019, 12(7), 861-883. DOI: 10.17516/1999-494X-0186.

(C) Siberian Federal University. All rights reserved

This work is licensed under a Creative Commons Attribution-NonCommercial 4.0 International License (CC BY-NC 4.0).

* Corresponding author E-mail address: pokhabov_yury@mail.ru 


\title{
Проектирование высокоответственных систем
}

\author{
с учетом надежности \\ на примере поворотной штанги
}

\author{
Ю.П. Похабов \\ $A O$ «НПО ПМ МКБ»
}

Россия, 662972, Железногорск, ул. Ленина, 55a

Статья посвящена одной из технологий проектирования высокоответственных систем на примере поворотной итанги космического аппарата, которая позволяет учитывать заданные требования к надежности с самых первых конструкторских решений. Предложен алгоритм построения обобщенной параметрической модели функционирования изделий, которая предназначена для проведения расчетной и экспериментальной верификации выходных параметров функиионирования и соотнесения их с заданными показателями надежности. Приведена методика конструкторско-технологического анализа надежности, позволяющего прочзводить аналитическую верификацию параметров функционирования на предмет выявления необходимых и достаточных требований к проведению расчетноэкспериментальной верификаџии и изготовлению изделий с заданной надежностью. Данная технология проектирования, основанная на параметризации и проведении конструкторскотехнологического анализа надежности, позволяет достигать безотказности механических устройств раскрытия whp $R \equiv 1$ вне зависимости от типа серийного производства. При этом потенциальные отказы могут быть с успехом устранены или смягчены на самых ранних стадиях жизненного ичикла (проектировании и конструировании), а возможные риски возникновения отказов - своевременно оченены.

Ключевые слова: проектирование, проектирование с учетом надежности, высокоответственные системы, космический аппарат, конструкторско-технологический анализ надежности (КТАН).

Введение. Согласно существующей концепции космических полетов космические аппараты (КА) после выведения на орбиту должны отделиться от ракеты-носителя и раскрыть все свои складные конструкции (панели солнечных батарей, антенны, штанги приборов). Отделение КА и раскрытие его складных конструкций производятся с помощью механических устройств одноразового срабатывания. Такие механизмы обеспечивают складывание конструкций в заданном объеме, удержание их в сложенном положении с требуемой прочностью и жесткостью, отделение от опорного основания по заданной команде, организованное раскладывание и фиксацию в раскрытом положении, а в случае отделения вместо раскладывания и фиксации разведение на безопасное расстояние КА и последней ступени ракеты-носителя. При этом любой их отказ способен привести к гибели аппарата (Sinosat-2, Канопус-СТ и др.), существенному ограничению его функциональных возможностей (Telstar 14R, Ресурс-П № 3 и др.) либо снижению срока активного существования из-за незапланированного расхода топлива двигателей коррекции на спиновые маневры для создания динамических воздействий на заклинившие механизмы («ANIK E2», «SES-4» и др.). Поэтому одноразовое срабатывание механических устройств КА должно происходить с безотказностью не ниже 0,9995, а фактически - whp $R \equiv 1$.

В условиях единичного (мелкосерийного) типа производства, характерного для изготовления КА, из-за финансово-экономических ограничений невозможно подтвердить вероятностно- 
статистическими методами теории надежности достоверность показателей безотказности вблизи единицы. Вследствие этого надежность срабатывания механических устройств на практике обеспечивают рациональным выбором выходных параметров физических процессов при функционировании путем соблюдения определенных требований выполнения проектноконструкторских работ и установленных правил проведения аналитической, расчетной и экспериментальной верификации [1]. Причем не существует каких-либо методик, позволяющих не только соотнести выходные параметры функционирования с заданными показателями вероятности безотказной работы, но и выявить все необходимые и достаточные параметры функционирования, влияющие на надежность.

Это приводит к тому, что, как показывает статистика отказов механических устройств раскрытия на отечественных и зарубежных КА за период 2009-2016 гг., их средняя безотказность на практике не превышает значений 0,989-0,996 [2]. Из анализа причин отказов известно, что они могут быть вызваны внезапным исчезновением зазоров в кинематических парах (Кику-8, Союз ТМА-17M), редким сочетанием производственных факторов (Intelsat-19), технологическими дефектами (Канопус-СТ, Прогресс М-19M), попаданием в механизм раскрытия посторонних предметов (Skylab, Telstar 14, Telstar 14R), отказами приводов раскрытия (EchoStar-4), преждевременным раскрытием (Ресурс-П № 3), конструкторско-технологическими факторами (Маяк), холодной сваркой (Galileo) и т.д. Практически все приведенные примеры причин отказов относят их к категории редких. Причем среди причин отказов почти не встречаются отказы из-за нарушения условий прочности и запасов движущих моментов (сил), поскольку их обеспечение является основой расчетной и экспериментальной верификации механизмов [3].

Так как конечной целью любой разработки является обеспечение заданной надежности (никому не нужны внезапно отказывающие изделия), существует объективная необходимость в разработке новых методик анализа и оценки надежности высокоответственных систем (которые в принципе не должны отказывать). Для этого необходимо научиться выявлять редкие потенциальные отказы на максимально ранних стадиях выполнения проектно-конструкторских работ, когда ошибки еще можно исправить без привлечения значительных незапланированных ресурсов на их исправление (согласно правилу десятикратных затрат). Важно, чтобы такие методики были неотъемлемой частью технологии проектирования, направленной на своевременную оценку и выбор конструкторских решений для обеспечения заданных требований надежности.

Конструкторско-технологический анализ надежности. Идея проведения анализа надежности с учетом конструкторских и технологических факторов проектирования исходит из того, что, по данным различных источников, доля конструкторских и технологических ошибок в причинах отказов составляет до $85 \%$ [2].

Согласно методике расчета надежности функционирования механических устройств раскрытия КА на этапе проектирования, изложенной в работе [3] и являющейся основой обеспечения надежности механических устройств раскрытия в АО «ИСС» имени академика М.Ф. Решетнева» [4], формула для расчета надежности в запас (в предположении, что составляющие формулы независимы по надежности) имеет вид

$$
R=R_{\text {пр }} R_{\phi}
$$


где $R_{\text {пр }}, R_{\phi}$ - надежность по прочности и функционированию.

С учетом использования конструктивных запасов формулы для определения надежности по прочности и функционированию (1) можно преобразовать к виду

$$
\begin{aligned}
& R_{\text {пр }}=P(N / n>f S), \\
& R_{\phi}=P\left(M_{\text {дв }}>k M_{\text {с }}\right),
\end{aligned}
$$

где $N, S, M_{\text {дв }}, M_{\text {с }}$ - соответственно несущая способность, действующие нагрузки, момент движущих сил и момент сил сопротивления; $n$ - запас прочности конструкционных материалов; $f$ - коэффициент безопасности; $k$ - коэффициент запаса движущего момента, отражающий идею о необходимости разделения средних значений движущего момента и моментов сил сопротивления для повышения надежности.

Поскольку в основе расчетно-экспериментального метода определения надежности лежит обоснование конструкторских и технологических решений (которые детализируют проектные решения и учитывают заводские технологии изготовления), формула (1) на этапе разработки рабочей конструкторской документации (привязанной к технологическим возможностям производства):

$$
R=R_{\text {пр }} R_{\phi} R_{\text {кт }}
$$

где $R_{\text {кт }}$ - конструкторско-технологическая надежность, которая помимо обеспечения заданных условий конструкционной прочности и запасов движущих моментов раскрывающих приводов зависит от конструкторских и технологических факторов, связанных, например, с обеспечением зазоров в кинематических парах, вибростойкостью соединений, стабильностью настроек механизмов, достаточностью хода исполнительных устройств, режимами выполнения особо ответственных операций и т.д.

Очевидно, что в случае $R_{\text {кт }}=1$ формула (4) приводится к (1), а при $R_{\text {пр }} \equiv 1$ и $R_{\phi} \equiv 1$ выражение принимает вид

$$
R=R_{\text {Кт }} \leq 1
$$

Выражение (5) вытекает из формулы полной вероятности

$$
R(t)+Q(t)=1
$$

где $R(t)$ - функция надежности, $R(t)=P(\tau>t) ; Q(t)-$ функция отказа, $Q(t)=P(\tau<t)$.

На основании только формул (1)-(3) можно сделать вывод, что заданный уровень безотказности по проектным параметрам прочности и функционирования обеспечен, т.е. $R>\underline{R}$, но невозможно предоставить объективных доказательств того, что

$$
Q(t)<1-\underline{R}
$$

Из сопоставления формул (5)-(7) следует

$$
R_{\mathrm{KT}}=1-Q(t)
$$


Таким образом, расчетно-экспериментальный метод определения проектной надежности $[3,4]$ применительно к высокоответственным механическим устройствам раскрытия по условиям прочности и запасам движущих моментов является недостаточным без учета конструкторско-технологических факторов. Поэтому для высокоответственных устройств необходимо применять технологии проектирования, позволяющие своевременно исключать или смягчать конструкционные ошибки, способные привести к потенциальным отказам, добиваясь обеспечения условия $R_{\text {кт }} \rightarrow 1$.

Экспертиза проектно-конструкторских и конструкторско-технологических разработок механизмов срабатывания на практике методом конструкторско-технологического анализа надежности (КТАН) показывает, что разработки, прошедшие установленную в ракетнокосмической отрасли процедуру расчетной и экспериментальной верификации, а в некоторых случаях даже летную квалификацию, продолжают содержать конструкторские и технологические ошибки в технической документации, не позволяющие надеяться на выполнении заданных требований надежности выше значений 0,999 [2].

Для обоснования конструкторско-технологического подхода к анализу надежности рассмотрим процесс проектирования (конструирования) как обобщенную модель параметрического функционирования изделия для обеспечения заданной надежности.

Модели для обоснования конструкторско-технологического анализа надежности. Концептуальная модель. Надежность по ГОСТ 27.002-2015 определяется как свойство объекта сохранять во времени способность выполнять требуемые функции в заданных режимах и условиях применения, технического обслуживания, хранения и транспортирования (или просто эксплуатации). Таким образом, когда мы говорим о надежности, то имеем в виду:

• определенный перечень требуемых функиий, обусловленных критериями, которые могут быть установлены;

- естественный ход времени работы объекта или наработки, когда должно быть обеспечено выполнение требуемых функций;

- заданные режимы и условия эксплуатации, в которых требуемые функиии не могут быть нарушены.

Из сказанного следует, что надежность можно представить как проявление объектом требуемых функций при определенных характеристиках времени, режимов и условий эксплуатации. Если под моделью согласно ГОСТ Р 54136-2010 понимать абстрактное описание реальности в любой форме (включая математическую, физическую, символическую, графическую или описательную), которая представляет определенный аспект этой реальности, то приведенное представление о надежности есть не что иное, как концептуальная модель.

Такая модель позволяет перейти к структуре моделируемой системы в виде взаимосвязанных понятий: надежность $(R) \rightarrow$ требуемые функиии $(f) \rightarrow\{$ время $(t)$, режимы и условия эксплуатации $(m)\}$ и отношений между ними:

$$
R \Rightarrow f(t, m) .
$$

Выражение (9) определяет согласованность элементов концептуальной модели между собой, но не дает представления о математических зависимостях, которые позволяют характеризовать надежность как физическое свойство объекта.

$$
-865-
$$


Понятийная модель. Понятийная модель надежности определяет термины понятий, составляющих концептуальную модель, и связь между ними, что является информационной основой для разработки моделей по определению количественных показателей надежности.

Ключевой основой понятийной модели надежности служит понятие «функция». Согласно ГОСТ Р 53394-2009 под функцией понимают описание (как правило, вербальное) служебного назначения изделия, т.е. того, что должно делать изделие (составная часть) при его применении (использовании). В Большой советской энциклопедии функция определяется как отношение двух (группы) объектов, в котором изменение одного из них ведет к изменению другого. Наконец, в разрабатываемой в настоящее время группе стандартов серии «Системы и комплексы космические» термин «функция» трактуется как реализация объектом выходного эффекта. Таким образом, под функцией понимаем:

- атрибут объекта;

- результат входного воздействия на выходе процесса;

- выходную характеристику объекта при выполнении служебного назначения.

Согласно ГОСТ 27.002-2015 требуемые функции могут быть установлены критериями в виде, например, задания для каждой функиии набора параметров, характеризующих способность ее выполнения, и допустимых пределов изменения значений этих параметров. В этом случае требуемые функции имеют вид $f=\left\{f_{i} \mid \forall i=(\overline{1, n})\right\}$, что с учетом параметрических критериев: выходных параметров $X_{i} \mapsto f_{i}$ и допустимых диапазонов их изменения $\left[\underline{X}_{i}, \bar{X}_{i}\right]$, можно представить множеством

$$
f \Rightarrow\left\{X_{i} \mid \underline{X}_{i} \leq X_{i} \leq \bar{X}_{i}\right\}
$$

До начала эксплуатации (при наработке $t=0$ ) множество (10) представляет характеристику атрибута объекта, которую принято называть работоспособностью - состоянием объекта, в котором он способен выполнять требуемые функции. Очевидно, что с началом наработки при $t>0$ работоспособность объекта не должна изменяться, поэтому в процессе эксплуатации множество (10) принимает вид, зависимый от наработки:

$$
f \Rightarrow\left\{X_{i}(t) \mid \underline{X}_{i} \leq X_{i}(t) \leq \bar{X}_{i}\right\},
$$

Множество (11) можно выразить через допустимую область значений выходных параметров $D_{x}$

$$
D_{x}=\left\{X_{i}(t) \mid \underline{X}_{i} \leq X_{i}(t) \leq \bar{X}_{i}\right\} .
$$

Множества (11) или (12) есть не что иное, как характеристика преходящих состояний объекта, т.е. последовательно сменяемых состояний, характеризующих изменение требуемых функций $f$ во времени при его функиионировании - выполнения в объекте (системе) проиесса (процессов), соответствующего (соответствующих) заданному алгоритму и (или) проявление объектом заданных свойств (согласно определению термина «функционирование» по ГОСТ 22487-77). С учетом (12) требуемые функции $f$ можно рассматривать как выполнение объектом своего служебного назначения с выходными параметрами $X_{i}(t)$, которые не выходят за пределы допустимой области $D_{x}$. Как известно (в соответствии с ГОСТ Р ИСО 9000-2015), процесс является совокупностью взаимосвязанных и (или) взаимодействующих видов деятель- 
ности, использующих входы для получения намеченного результата. Таким образом, в процессе функционирования значения выходных параметров $X_{i}(t)$ обусловлены результатом входных (внешних) воздействий $V_{i}$ :

$$
\left[\underline{X}_{i}, \bar{X}_{i}\right] \stackrel{\cong}{\rightarrow}\left[\underline{V}_{i}, \bar{V}_{i}\right] .
$$

Допустимые диапазоны изменений выходных параметров всегда отражают по форме внешние воздействия $V_{i}$ и устанавливаются исходя из их предельных величин, но не во всех случаях строго соответствуют им. Это обусловливается неочевидными проявлениями поведения объектов вблизи границ диапазонов входных воздействий $\left[\underline{V}_{i}, \bar{V}_{i}\right]$, например в случаях резонанса, флаттера, автоколебаний, последствий технологической наследственности и прочих явлений, которые обусловлены взаимным влиянием друг на друга режимов и условий эксплуатации. Для определения случаев, влияющих на ограничения или потерю работоспособности объектов при неблагоприятных сочетаниях режимов и условий эксплуатации, необходимо иметь детальное представление о сути этих понятий.

Дефиниции «режим» и «условия» имеют близкие, но различные по смыслу значения. Под режимом эксплуатации можно понимать условия, которые присущи техническому объекту независимо от того, наблюдаются какие-либо внешние воздействия или эти воздействия отсутствуют. В то же время согласно ГОСТ 26883-86 под внешними воздействующими факторами понимается совокупное воздействие явлений, процессов или среды, внешних по отношению к изделию или его составным частям, которые вызывают или могут вызвать ограничение или потерю работоспособного состояния изделия в проиессе эксплуатаџии. Таким образом, при функционировании следует различать внутренние условия, присущие объекту (локализованные в нем), и внешние условия, навязанные окружающими средой по границам интерфейсов.

Внутренние условия соответствуют исключительно режимам эксплуатации исследуемого объекта и являются следствием воздействия на него, как правило, антропогенных факторов до начала эксплуатации. Эти факторы порождаются людьми при проектировании, конструировании, выборе и использовании технологий, изготовлении, технической приемке и приемочных испытаниях; к их числу относятся, например, алгоритмы функционирования, структура и конструкция технического объекта, процедуры сборки, настройки и регулировки механизмов, состояние объекта после изготовления, факторы технологической наследственности и т.п. По всей видимости, именно совокупность внутренних условий (установленный порядок функционирования и конструкторско-технологическая наследственность) в общей теории надежности механических систем В.В. Болотина следует понимать под «пространством качества», которое включает свойства, присущие системе, заданные технологические, эксплуатационные и прочие требования [5].

Внешние условия соответствуют совокупному действию режимов эксплуатации внешних технических объектов (техногенным факторам) и факторов природной среды относительно интерфейсов исследуемого объекта. Если исходить из аналогий, то совокупность внешних режимов и условий эксплуатации на границах интерфейсов В.В. Болотин определяет как «пространство входных параметров», понимая под этим факторы внешней среды.

Приведенное понимание режимов и условий позволяет рассматривать, например, ракетуноситель с выводимым КА как техническую систему, в которой на космический аппарат дей-

$$
-867-
$$


ствуют внешние воздействия со стороны ракеты-носителя и внешней природной среды, например, в виде акустических волн, генерируемых обшивкой головного обтекателя в набегающем воздушном потоке, и это воздействие сочетается с режимами эксплуатации самого аппарата, например с частотами собственных колебаний его элементов конструкции, вызывая ограничения на выходные параметры вибропрочности из-за резонансов. Если рассматривать только функционирование космического аппарата, то примерами режимов и условий его эксплуатации могут служить следующие явления:

- режимы (внутренние условия) - это ударные нагрузки при срабатывании пиротехнических устройств замков зачековки, процессы развертывания складных конструкций, включая динамические нагрузки при фиксации в рабочем положении, тепловыделение приборов, жесткость конструкции (частота собственных колебаний);

- внешние условия - это вибрации ракетных двигателей (режим их работы), динамические воздействия в результате расцепки ступеней ракеты-носителя (режимы переходных процессов), ударные нагрузки при отделении от ракеты-носителя (режимы отделения), действие факторов космического пространства (природная среда космоса) после сброса головного обтекателя и пр.

При эксплуатации внутренние и внешние условия накладываются друг на друга, усиливая или ослабляя входные воздействия $V_{i}$ в соответствии с физическими законами природы, что приводит к ограничениям на выполнение объектом требуемых функций, например:

- суммирование предварительно-напряженного состояния элементов конструкции с напряженно-деформированным состоянием от внешних нагрузок (в соответствии с принципом суперпозиции) приводит к снижению несущей способность конструкции;

- геометрическая нестабильность углепластиковых конструкций из-за технологической наследственности при совместном действии тепловых и радиационных потоков космического пространства приводит с течением времени к снижению точности орбитального позиционирования рабочих поверхностей конструкций;

- механическая вибрация при работе ракетных двигателей в сочетании с вибрационными перемещениями (подвижностью под действием вибрации номинально неподвижных деталей машин) приводит к самоотвинчиванию (ослаблению затяжки) резьбовых соединений;

- возрастание внутреннего давления в герметичных емкостях при падении внешнего давления и увеличении температуры окружающей среды приводит к снижению несущей способности силовой конструкции емкости и уменьшению ее ресурса по герметичности;

- при раскрытии складных конструкций в зависимости от аномально низкой или высокой температуры внешней среды резко меняются физико-механические свойства материалов конструкций, что приводит к существенным изменениям сопротивления движению в шарнирах, в результате может снизиться несущая способность конструкции (при малом сопротивлении вследствие высокой температуры) либо надежность раскрытия (при большом сопротивлении вследствие низкой температуры).

$$
-868-
$$


Таким образом, при установлении допустимых границ выходных параметров $X_{i}(t)$, обусловливающих выполнение требуемых функций $f$, необходимо рассматривать взаимодействие внутренних и внешних условий в результате входных воздействий $V_{i}$, которые определяются режимами и условиями эксплуатации $m$ при их наложении. Для стационарных стохастических процессов минимальные и максимальные реактивные эффекты на входные воздействия можно выразить в следующем виде:

$$
\begin{aligned}
& \underline{V_{i}}=\alpha_{i}=\operatorname{argmin}_{m} V_{i}(m) \Leftrightarrow \underline{V}_{i}(m)=V_{i}\left(\alpha_{i}\right), \\
& \bar{V}_{i}=\beta_{i}=\operatorname{argmax}_{m} V_{i}(m) \Leftrightarrow \bar{V}_{i}(m)=V_{i}\left(\beta_{i}\right) .
\end{aligned}
$$

С учетом выражений (14)-(15) соотношение (13) можно записать как

$$
\left[\underline{X}_{i}, \bar{X}_{i}\right]=\left[\alpha_{i}, \beta_{i}\right]
$$

при этом требуемые функции (11) с учетом режимов и условий эксплуатации принимают вид

$$
f \Rightarrow\left\{X_{i}(t) \mid \alpha_{i} \leq X_{i}(t) \leq \beta_{i}\right\}
$$

и, соответственно, область допустимых значений выходных параметров $D_{x}(12)$

$$
D_{x}=\left\{X_{i}(t) \mid \alpha_{i} \leq X_{i}(t) \leq \beta_{i}\right\} .
$$

Согласно представленной понятийной модели надежность (9) с учетом (14)-(18) можно выразить следующим образом:

$$
R \Rightarrow\left\langle X_{i}, \alpha_{i}, \beta_{i}, t\right\rangle
$$

Модель единства целей разнотипных компонентов. Для определения перечня выходных параметров $X_{i}(t)$ в выражениях (17)-(19) используют модель единства целей разнотипных элементов при функционировании объектов. Как известно, любой сложный технический объект состоит из различных по природе (принципу действия) компонентов (элементов, узлов, подсистем, систем, технологий, сырья различного происхождения, алгоритмов, программных компонентов и т.д.), которые реализуются одновременно при достижении цели своего служебного назначения. Если каждый из компонентов может применяться отдельно (независимо), а одновременное их использование и взаимодействие не вызывает отказ по общей причине, то модель функционирования такого объекта может быть представлена вектор-столбцом выходных параметров $X_{i}$ критичных элементов, недопустимое изменение значений которых способно вызвать отказ объекта по собственной причине, присущей природе соответствующего физического явления,

$$
\left\{X_{i}\right\}=\left(M, L, T, W_{t}, W_{i}, W_{v}, \ldots\right)^{\mathrm{T}},
$$

где $M, L, T, W_{t}, W_{i}, W_{v}, \ldots$ - соответственно, вещественные, пространственные, временны́е, тепловые, электрические, механические и иные выходные параметры критичных элементов технического объекта, место которых обозначено многоточием.

Таким образом, вектор-столбец (20) - представление технического объекта с позиций выходных параметров его критичных элементов, каждый из которых может иметь различную природу физических явлений.

$$
-869-
$$


Модель фиктивных элементов. Если одни и те же критичные элементы в процессе функционирования могут одновременно или последовательно находиться в различных предельных состояниях (например, по устойчивости, прочности, условиям осуществления кинематической подвижности, конструктивно-технологическим ограничениям и т.п.), необходимо использовать метод фиктивных элементов [3].

Фиктивным элементом называется реальный элемент, рассматриваемый лишь в одном из предельных состояний (имеющий лишь один вид отказов). Если тот же элемент может иметь и другой вид отказов, то вводится другой фиктивный элемент, находящийся в другом предельном состоянии. Число фиктивных элементов соответствует предельным состояниям, в которых может находиться реальный элемент при переходе его из одного состояния в другое. Представление реального элемента может быть сведено к виду

$$
\left\{X_{j}\right\}=\left(X_{j}^{1}, X_{j}^{2}, \ldots, X_{j}^{h}\right)^{\mathrm{T}} \forall h=(\overline{1, m}) ; j=\text { const } \in \mathbb{N},
$$

где $h$ - число предельных состояний, в которых может находиться $j$-й критичный элемент.

Фиктивные элементы (21), которые могут находиться в разных предельных состояниях, должны быть учтены при составлении вектор-столбца (20) и рассмотрены наравне с реальными элементами, которые находятся лишь в одном предельном состоянии. С учетом (20)-(21) вектор-столбец выходных параметров $X_{i}$ критичных элементов можно представить в обобщенном виде:

$$
\left\{X_{i}\right\}=\left(X_{1}, X_{2}, \ldots, X_{i}\right)^{\mathrm{T}} \forall i=(\overline{1, n}) .
$$

Вектор-столбец (22) характеризует функциональность объекта, которая согласно ГОСТ 28806-90 (применительно к техническим объектам) понимается как совокупность свойств, определяемых наличием и конкретными особенностями набора функиий, способных удовлетворять заданные или подразумеваемые потребности.

Для выполнения требуемых функций $f$ должна быть обеспечена возможность каждому из параметров функционирования критичных элементов в (22) находиться в допустимом диапазоне, соответствующем предельному состоянию, выявленному в работах [2, 3]. С учетом (16) предельные состояния критичных элементов определяются формулой

$$
X_{i \text { пред }}=\left\{\begin{array}{l}
X_{i}=\alpha_{i} \\
\bar{X}_{i}=\beta_{i}
\end{array} .\right.
$$

Предельные значения выходных параметров $X_{i}$ критичных элементов (23) находят с помощью моделей отказов, использующих различные схемы соотношений входов-выходов процессов, максимально соответствующих представлениям и знаниям о физических законах функционирования объекта.

Модели отказов. Схема «параметр-допуск» описывает универсальную физическую модель отказов, когда случайные изменения выходных параметров могут быть ограничены заданным двусторонним допуском $X_{i} \in\left[\alpha_{i}, \beta_{i}\right]$. В этом случае отказ определяется по формуле $\forall X_{i}=\left[\left(X_{i}<\alpha_{i}\right) \vee\left(X_{i}>\beta_{i}\right)\right]$.

При использовании модели отказов по схеме «параметр-допуск» между собой соотносят параметры с одинаковой размерностью величин, которые могут быть измерены и рассчитаны 
на основе физических законов природы. Выполнение требуемых функций при функционировании по схеме «параметр-допуск» обеспечивается в случае, если

$$
\alpha_{i} \leq X_{i}(t) \leq \beta_{i}
$$

Неравенство (24) можно также выразить в безразмерном виде относительно результата входного воздействия $V_{i}$ на выходе процесса как вероятность осуществления желательного события $P_{i}$ :

$$
P_{i}=P\left[\alpha_{i} \leq X_{i}(t) \leq \beta_{i}\right]
$$

Если выходной параметр $X_{i}$ рассматривается в стохастической постановке, когда значения случайных величин группируются по определенному закону вблизи наиболее вероятных и неизменных во времени величин с плотностью распределения $\varphi(X)$, например в случае нормального распределения Гаусса, усеченного с двух сторон, то выражение безотказности при функционировании (25) будет иметь вид

$$
P_{i}=F\left(\gamma_{\mathrm{H}}\right)+F\left(\gamma_{\mathrm{B}}\right)-1 \text {, }
$$

здесь $\gamma_{\mathrm{H}}=\left(m_{X_{i}}-\alpha_{i}\right) / S_{X_{i}} ; \gamma_{\mathrm{B}}=\left(\beta_{i}-m_{X_{i}}\right) / S_{X_{i}}$;

где $F\left(\gamma_{\mathrm{H}}\right), F\left(\gamma_{\mathrm{B}}\right)$ - табулированные функции нормального распределения; $m_{X_{i}}, S_{X_{i}}$ - математическое ожидание и среднее квадратическое отклонение параметра $X_{i}$.

Физический смысл установления границ изменения выходных параметров $X_{i}$ в стохастической постановке заключается в разнесении математических ожиданий этих параметров с параметрами входных воздействий на величину, обеспечивающую достижение требуемой надежности. Усечение плотности распределения $\varphi(X)$ можно рассматривать как область пересечения плотностей распределения входных и выходных параметров, площадь которой стремится к ничтожно малой величине (близкой к нулю). В детерминированной постановке это достигается установлением соответствующих значений коэффициентов $k_{\alpha_{i}}=V_{i} / \alpha_{i}$ и $k_{\beta_{i}}=\beta_{i} / V_{i}$, что следует из соотношений (14)-(15) при сужении области допустимых значений выходных параметров. В прочности такие коэффициенты известны как коэффициенты безопасности и запасы прочности.

С учетом двустороннего допуска на вероятность достижения выходных параметров (один из которых по определению равен единице) выражения (25)-(26) можно представить в виде

$$
\underline{P}_{i} \leq P_{i} \leq 1
$$

Чаще всего модель отказов по схеме «параметр-допуск» на практике есть смысл рассматривать как частный случай модели отказов по схеме «нагрузка-сопротивление», предполагая, что двойное неравенство (24) может распадаться на односторонние неравенства, например, если нижняя и верхняя границы выходного параметра определяются разными по своей сути физическими явлениями. Так при развороте однозвенной штанги КА нижняя граница движущего момента на приводе находятся сопротивлением повороту штанги (надежностью раскрытия), а верхняя - прочностью штанги в момент фиксации в рабочем положении (когда избыточная кинетическая энергия движущейся штанги переходит в потенциальную энергию ее деформации). В первом случае под нагрузкой понимаем момент сил сопротивления пово- 
роту, а под сопротивлением - движущий момент на приводе. Во втором случае под нагрузкой понимаем движущий момент на приводе, а под сопротивлением - прочность штанги на изгиб.

Безотказное функционирование по схеме «нагрузка-сопротивление» обеспечивается в случае, если

$$
X_{i}(t)>\alpha_{i}
$$

Неравенство (28) по аналогии с (25) можно также выразить в безразмерном виде:

$$
P_{i}=P\left[X_{i}(t)>\alpha_{i}\right]
$$

Если выходной параметр $X_{i}$ рассматривается в стохастической постановке при нормальном распределении Гаусса усеченном слева, то выражение (29) таково:

$$
P_{i}=F\left(\gamma_{\mathrm{H}}\right)
$$

С учетом двустороннего допуска на вероятность достижения выходных параметров выражения (29)-(30) можно представить в виде (27).

В случаях, когда невозможно определить физические параметры, приводящие к отказам, из-за неочевидности причинно-следственных взаимосвязей процессов, которые происходят при функционировании внутри системы, применяют модель отказов по схеме «черный ящик».

Безотказное функционирование по схеме «черный ящик» выражается исключительно в безразмерном виде, как вероятность совершения случайных событий, которая может измеряться числом от 0 до 1. Причем с учетом замечаний, приведенных в ГОСТ Р 50779.10-2000, данное число может отражать как относительную частоту в серии наблюдений, так и степень уверенности в том, что некоторое событие произойдет. Фактическое значение вероятности совершения событий всегда меньше единицы. Для получения приемлемого уровня надежности используют резервирование (структурное или функциональное) критичных элементов.

Надежность по схеме «черный ящик» определяют относительно входного воздействия $V_{i}$ как результат наблюдений с вероятностью осуществления желательных исходов случайных событий $P_{i}$. Вычисление вероятности $P_{i}$ может быть произведено двумя способами, в зависимости от природы случайных величин:

1) если случайной величиной являются случайные события, например срабатывания устройств однократного применения (пирочек), то вероятность событий определяют по формуле

$$
P_{i} \approx n / N
$$

где $n$ - число неотказавших элементов; $N$ - число элементов, поставленных на эксперимент;

2) если случайной величиной является случайный процесс, который определяется временем до отказа (в общем случае - наработкой до отказа), то вероятность событий находят по формуле

$$
P_{i}=\int_{t}^{\infty} \varphi(t) d t,
$$

где $\varphi(t)$ - плотность распределения времени до отказа за время $t$.

$$
-872-
$$


С учетом двустороннего допуска на вероятность достижения выходных параметров выражения (31)-(32) можно представить в виде (27).

При возникновении случайных событий спорадического характера в виде неучтенных (непредвиденных) воздействий (чаще всего антропогенного характера) применяют модель отказов по схеме «события-проверки». Например, неустановка стопорения резьбового соединения это событие, которое при вибрациях, вызванных работой двигателей ракеты-носителя, может привести к самоотвинчиванию крепежных гаек и возникновению отказов механизмов КА: разрушению резьбового соединения или к нарушению регулировок механизма и вследствие этого к нештатному срабатыванию (раскрытию под головным обтекателем) или несрабатыванию при штатном раскрытии. Предупреждение отказов (событий) в приведенном примере обусловливается только осуществлением контроля (проверок) соответствующих мест стопорения резьбовых соединений. Типичные примеры причин отказов по схеме «события-проверки»зацепление раскрывающихся элементов за смежные конструкции, попадание в механизмы раскрытия посторонних предметов (клея), грубые нарушения технологии проведения сборочных работ и т.п.

Безотказное функционирование по схеме «события-проверки» может быть выражено в безразмерном виде с помощью функции, заданной на двухэлементном множестве $\{0,1\}$ и принимающей значения в том же множестве (символом 0 обозначается ложное высказывание, а символом 1 - истинное). Модель отказов по схеме «события-проверки» отражает принцип спорадичности осуществления событий, при этом меры по предупреждению отказов подчиняются методам алгебры логики. Для описания отказов по схеме «события-проверки» используется выражение

$$
P_{i}= \begin{cases}1 & \text { событие, }, \text { способное вызвать отказ, не произошло, } \\ 0 & \text { событие, способное вызвать отказ, произошло. }\end{cases}
$$

Применяя модель единства целей разнотипных компонентов (20) с учетом моделей фиктивных элементов (21) и различные схемы отказов в зависимости от представления о физической природе явлений (24)-(33), происходящих при выполнении требуемых функций, можно построить область допустимых значений выходных параметров $D_{x}(18)$. Эта область безразличным образом может состоять из выходных параметров в виде физических величин $X_{i}(t)$ с границами изменения $\left[\alpha_{i}, \beta_{i}\right](24)$ и (28) или безразмерных согласованных показателей в виде вероятностей событий $P_{i}$ с границами изменения $\left[\underline{P}_{i}, 1\right](25)-(26)$ и (29)-(32), либо $[1,1]$ - в случае создания условий для выполнения требуемых функций при спорадичном проявлении отказов (33). Отсюда следует, что физические модели отказов (схемы «параметр-допуск» и «нагрузка-сопротивление») могут быть сведены к стохастическим моделям отказов (схемы «черный ящик» и «события-проверки»), а стохастические модели отказов, в свою очередь, - к физическим моделям отказов при условии получения необходимых и достаточных знаний об основах физических процессов при выполнении требуемых функций.

Модель расчета надежности. С учетом (9)-(33) область допустимых значений выходных параметров $D_{x}(18)$ образована пространством выходных параметров $X_{i}$, а также пространствами качества и входных параметров в виде $\alpha_{i}$ и $\beta_{i}$. Очевидно, что переход выходных параметров 
за пределы границ области их допустимых значений приводит к отказу, отсюда следует формула надежности, известная из работы [5]:

$$
R=P\left\{X_{i}(t) \in D_{x} ; t \in\left[0, t_{\mathrm{K}}\right]\right\},
$$

Формула (34) является детализированным отображением представления о надежности (9) и (19), пригодной для получения ее количественной оценки. Причем если область допустимых значений $D_{x}$ образована выходными параметрами, приведенными к согласованному безразмерному виду, который определяется вероятностью совершения требуемых событий при функционировании, то границы этой области могут быть сведены к интервалу $D_{x} \in[0,1]$. Тогда при последовательном соединении критичных элементов (22), если они независимы в смысле надежности, вместо (34) можно использовать широко известную формулу

$$
R=\prod_{i=1}^{n} P_{i} .
$$

Обобщенная параметрическая модель функционирования. Из рассмотренных моделей вытекает обобщенная параметрическая модель функционирования технических объектов, позволяющая представить выходные характеристики в виде:

- функциональности (совокупности свойств, определяемых наличием и набором возможностей выполнять требуемые функции) с учетом моделей единства целей разнотипных компонентов и фиктивных элементов в виде (22);

- работоспособности (состояния, в котором объект способен выполнять требуемые функции) с учетом понятийной модели в виде (18);

- надежности (свойства сохранять во времени выполнение требуемых функций в заданных режимах и условиях эксплуатации) с учетом модели расчета надежности в виде (34) или (35) - в случае представления параметров в согласованном безразмерном виде через вероятности совершения требуемых событий при функционировании.

Реализация обобщенной параметрической модели функционирования на практике осуществляется с помощью методики КТАН, состоящей из последовательного набора методов анализа и оценки надежности, которые используют непосредственно при проектировании или конструировании. Применение КТАН позволяет производить обоснование конструкторских решений с учетом заданной надежности.

Метод построения параметрической модели функционирования. Декомпозиция. Анализ надежности механических устройств раскрытия начинается с разбивки конструкции на конечное число составных частей методом декомпозиции так, чтобы каждая из составных частей подчинялась выполнению только одной из функциональных задач в рамках обеспечения раскрытия.

Цель декомпозиции - расчленить конструкцию механических устройств на конструктивные узлы, простейшие механизмы, функциональные группы и интерфейсы до уровня элементов, в которых реализуется режим точки единичного отказа. Каждый из таких элементов является потенциально критичным, если в нем не предусмотрена требуемая степень структурного или функционального резервирования. Например, пироузел следует декомпозировать до уровня отдельных пирочек и электрического интерфейса между пирочекой и бортовым комплексом

$$
-874-
$$


управления КА. Для механизма однозвенной поворотной штанги одной из точек единичного отказа является шарнирный узел (ШУ) поворота, место в конструкции штанги, где не предусматривается резервирования.

Анализ потенциальных отказов. Для каждой точки единичного отказа производится вербальное описание конструктивных элементов, характеризующих выполнение ими функций, которые соответствуют служебному назначению при изменении событий и смене состояний конструкции и механизмов в течение жизненного цикла. Например, ШУ поворотного механизма штанги в сложенном и рабочем положениях обеспечивает выполнение условий прочности, а при раскрытии - условий ее перемещения из сложенного положения в рабочее.

Далее выполняется вербальное описание отказов как гипотетических ситуаций, препятствующих выполнению рассматриваемых функций. Например, отказами ШУ могут быть структурное разрушение конструкционного материала или невозможность совершения относительных поворотов.

Анализ худшего сочетания факторов. Данный анализ по принципам проведения близок к широко используемому анализу худшего случая [6], который предназначен для исследования поведения объекта в наихудших условиях работы. Однако цель анализа худшего сочетания факторов заключается не в количественной оценке изменения значений выходных параметров объекта исследования, а в установлении комбинаций факторов худших входных воздействий (внешних режимов и условий) и режимов эксплуатации объекта (внутренних факторов), способных привести к отказам критичных элементов (нарушению выходных характеристик). Определение худших комбинаций производится как вербальное описание и служит исходными данными для выявления потенциальных причин отказов. В некоторых случаях худшим случаем может оказаться всего один воздействующий фактор, например внешняя нагрузка (квазистатическая, вибрационная, ударная, динамическая), или же комбинация факторов, когда к внешней нагрузке могут быть добавлены режимы эксплуатации объекта (например, статическая нагрузка, определяемая условиями выполнения сборочно-монтажных операций) и внешние (например, тепловые, электромагнитные) воздействия.

В частности, разрушение ШУ может быть вызвано сочетанием максимальной нагрузки и минимальной возможности конструктивного элемента сопротивляться ей (неудачный выбор конструкционного материала и/или назначение геометрических размеров и допусков поперечного сечения); а невозможность совершения относительных поворотов в шарнире поворотной конструкции может быть вызвана сразу несколькими различными причинами:

- запрессовкой шарнира как результата худшего сочетания радиальных зазоров после изготовления, толщины твердой смазки, механических нарушений условий трибосопряжения и теплового воздействия внешней среды;

- заклиниванием из-за худшего сочетания осевых зазоров и тепловых перемещений элементов конструкции;

- торможением из-за худшего сочетания движущего момента на приводе и моментов сил сопротивления в шарнире при аномально низкой температуре;

- невключением привода раскрытия из-за отказа вследствие, например, производственного брака комплектующих его деталей и сборочных единиц;

$$
-875-
$$


- зацеплением поворотной конструкции из-за ошибок проектирования, в результате которых на пути движения неожиданно возникает какое-либо препятствие.

Парирование возможных причин отказов. Знание потенциальных причин отказов позволяет определить условия, которые способны сделать выявленные причины отказов невозможными. Поиск таких условий производится методом отрицательных суждений (антитезисов). Логическая схема анализа построена на том, что в качестве исходного используется предвзятое суждение, основанное на том, что отказ какого-либо критичного элемента уже «произошел». Если при конструировании предприняты и документально подтверждены необходимые и достаточные меры, которые позволяют устранить причину возможного отказа, то это служит доказательством того, что означенное отрицательное суждение ложно и, следовательно, условие безотказности обеспечено. Под условием безотказности понимается свойство конкретного критичного элемента, делающее причину отказа невозможной.

Таким образом, любая возможная причина отказа может парироваться приданием критичным элементам строго определенных свойств, которые должны быть реализованы в конструкторской документации и осуществлены при изготовлении.

Например, для парирования отказов ШУ поворотной конструкции необходимо:

- обеспечить необходимую несущую способность шарнира, исключающую отказы по условиям прочности;

- установить значения радиальных зазоров в шарнире, которые при худших сочетаниях толщины смазки, возможных механических нарушениях трибосопряжений и тепловых воздействиях не вызывают запрессовки шарнира;

- установить осевые зазоры в шарнире больше, чем возможные относительные тепловые деформации конструкции в заданных условиях внешних тепловых воздействий;

- создать требуемый запас движущего момента на приводе, который обеспечивает устойчивое движение с заданными параметрами раскрытия;

- выбрать привод с требуемой вероятностью включения;

- доказать, что поворотная конструкция имеет траекторию движения, свободную от зацеплений за смежные конструкции или с учетом неконтролируемых положений нежестких конструктивных элементов в невесомости.

Выполнение процедуры парирования возможных причин отказов позволяет составить вектор-столбец (22) выходных параметров, которые должны быть присущи конструкции, чтобы исключить причины потенциальных отказов. Для ШУ поворотной штанги вектор-столбец параметров, характеризующих способность выполнять заданные функции, выглядит следующим образом:

$$
\{\mathrm{X}\}=\left(T, \Delta_{0}, \Delta_{s h}, M_{\text {дв }}, R_{d}, Q_{s t}\right)^{\mathrm{T}},
$$

где $T$ - несущая способность шарнира; $\Delta_{0}$ - радиальный зазор в шарнире; $\Delta_{s h}-$ осевой зазор в шарнире; $M_{\text {дв }}-$ движущий момент в шарнире; $R_{d}-$ надежность включения привода; $Q_{s t}-$ вероятность зацепления поворотной конструкции.

Чтобы обеспечить работоспособность шарнира, значения параметров, составляющих вектор-столбец (36), должны изменять свои значения в границах своих допустимых значений. 
Для определения допустимых границ выходных параметров необходимо на основании моделей отказов (24)-(33) построить параметрическую модель исследуемого объекта.

Параметризация. Поскольку параметры (36) количественно выражают свойства объекта, их значения определяются отношениями внутри объекта (режимами) с внешними объектами или средой (внешние режимы и условия). При этом отношения объектов (свойств) есть результат взаиморасположения, взаимосвязей и взаимодействия как внутри объекта, так и вне его [2]. В частности, параметры вектор-столбца (36) могут определяться следующими отношениями:

- $T$ - взаимодействием шарнира с внешними объектами и средой посредством нагрузок;

- $\Delta_{0}$ - взаиморасположением оси и проушины шарнира в радиальном направлении, их взаимосвязи через твердую смазку и взаимодействием с внешней средой при тепловом воздействием среды или при попадании в радиальный зазор абразивных частиц из окружающей среды;

- $\Delta_{s h}$ - взаиморасположением оси и проушины шарнира в осевом направлении и взаимодействием с внешней средой при тепловом воздействии;

- $M_{\text {дв }}$ - взаимосвязью с сопротивлением в шарнире и взаимодействием с температурой внешней среды, приводящей к увеличению сопротивления;

- $R_{d}$ - взаимосвязью резервирующих элементов в приводе;

- $Q_{s t}$ - взаиморасположением поворотной конструкции с внешними объектами.

Отношения свойств (объектов) могут быть выражены через физические величины либо вероятностные показатели в зависимости от принятой схемы процессов, моделирующих отказы. Модель отказов по схеме «нагрузка-сопротивление» целесообразно применять, если существует возможность использовать параметры, приводящие к отказам, которые могут быть получены путем проведения прямых измерений или вычислений. Исходя из этого схема «нагрузка-сопротивление» применяется для определения параметров $T, \Delta_{0}, \Delta_{s h}$ и $M_{\text {дв. Модель }}$ отказов по схеме «черный ящик» используется тогда, когда невозможно, сложно или нецелесообразно определять параметры, приводящие к отказам, например для привода, поступившего на сборку как покупной узел, работоспособность может быть оценена вероятностным показателем $R_{d}$, гарантируемым поставщиком. Модель отказов по схеме «события-проверки» целесообразно применять, когда отказы могут быть исключены путем принятия мер, делающих их невозможными, например, исключив все возможные причины зацеплений, можно утверждать, что $Q_{s t} \rightarrow 0$.

Таким образом, выражение (18) для описания области допустимых значений выходных параметров $D_{x}$ поворотной штанги может быть записано следующим образом:

$$
D_{x}=\left\{\begin{array}{c}
T>N \\
\Delta_{0}>0 \\
\Delta_{s h}>\Delta l \\
M_{\text {дв }>M_{\text {с }}} \\
R_{d} \geq R_{l i m} \\
Q_{s t} \rightarrow 0
\end{array}\right\},
$$

где $N$ - внешняя нагрузка на шарнир; $\Delta l$ - тепловые деформации конструкции вдоль оси шарнира; $M_{\mathrm{c}}$ - момент сопротивления в шарнире; $R_{\text {lim }}$ - заданная надежность привода.

$$
-877-
$$


Составление множества (18), которое с учетом рассматриваемого примера может принять вид (37), завершает процесс параметризации (параметрического моделирования) функционирования механического устройства раскрытия.

Проведение инженерного анализа надежности и составление зависимостей (36)-(37) позволяет выбрать все необходимые и достаточные параметры, влияющие на надежность функционирования, что делает возможной оценку надежности и создает условия для смягчения или исключения отказов.

Метод оценки теоретической надежности по проектным параметрам изделия. Расчет надежности по каждому из параметров, обеспечивающих требуемые функции, можно проводить обычными методами расчета по детерминированным или случайным величинам. При детерминированных расчетах надежности неопределенность параметров согласно схеме «нагрузка-сопротивление» представляется интервалом с верхней и нижней границами, а при расчетах по случайным величинам - в виде функции распределения случайных величин или процессов.

Для расчетов надежности механического устройства раскрытия, когда отказы не допускаются, расчеты надежности целесообразно проводить детерминированными методами, в результате чего с учетом (37) имеем

$$
\begin{gathered}
R_{1}=P(\underline{T}>\bar{N}) ; \\
R_{2}=P\left(\underline{\Delta}_{0}>0\right) ; \\
R_{3}=P\left(\underline{\Delta}_{s h}>\Delta \bar{l}\right) ; \\
R_{4}=P\left(\underline{M}_{\text {дв }}>\bar{M}_{\mathrm{c}}\right) ; \\
R_{5}=1-\left(1-R_{d}\right)^{p+1} ; \\
R_{6}=1-Q_{s t} .
\end{gathered}
$$

Каждая из составляющих оставляющих параметрической надежности (38) определяется с учетом обеспечения конструктивных запасов:

- резервированием путем $p$-й кратности структурного дублирования до выполнения условия $R_{5} \geq R_{\text {lim }}$;

- применением нормированных коэффициентов безопасности и запасов прочности до выполнения условия $R_{1}=P(T / \eta>f N)$;

- назначением запасов движущих моментов до выполнения условия $R_{4}=P\left(\underline{M}_{\text {дв }}>k M_{\mathrm{c}}\right)$;

- использованием параметрической избыточности в виде силовых и температурных развязок, например до выполнения условия $R_{3}=P\left(\underline{\Delta}_{s h}>\Delta \bar{l}\right)$ путем выбора конструктивных решений, обеспечивающих соотношения $\underline{\Delta}_{s h} \gg \Delta \bar{l}$ (если из двух шарниров, образующих единую ось вращения, один не имеет ограничений по перемещению вдоль оси);

- получением гарантированных результатов с использованием, например:

- минимаксных критериев до выполнения условия $R_{2}=P\left(\underline{\Delta}_{0}>0\right)$, когда соответствующие геометрические построения (3D-моделирование) и расчеты сделаны в запас путем минимизации максимально возможного риска;

- факторов инженерной психологии до выполнения условия $R_{6} \rightarrow 1$ с учетом критического анализа и минимизации последствия ошибок проектирования, способных привести к несанкционированным событиям.

$$
-878-
$$


Суммарная надежность с учетом $R_{1} \ldots R_{6}$ определяется по формуле (35). При соответствующем выборе конструктивных запасов для определения проектно-конструкторских параметров может быть достигнута надежность whp $R \equiv 1$. На практике это означает, что конструктор предоставил убедительные доказательства (провел все необходимые и достаточные расчеты и испытания) для обоснования выбора параметров изделия исходя из требований технического задания на разработку.

Однако, чтобы достичь предельной надежности на практике, необходимо убедиться, что при изготовлении и эксплуатации не будут совершены ошибки, способные нарушить обоснованность выбранных конструктором параметров, например из-за немотивированной замены конструкционных материалов, действий по умолчанию, нарушения технологий изготовления и т.д. Это становится возможным в том случае, если конструкторская документация, по которой произведена оценка теоретической надежности по проектно-конструкторским параметрам, будет без дополнительных толкований и разъяснений понятна не только лицам, которые непосредственно занимаются изготовлением и техническим контролем на производстве (не имеющим прямого отношения к конструкторскому замыслу), но и третьим лицам (экспертам).

Для реализации предельной надежности по завершению стадии выпуска рабочей конструкторской документации необходимо убедиться, что в ней установлены все необходимые и достаточные требования для обеспечения работоспособности и надежности, которые при изготовлении и эксплуатации должны быть выполнены, и подтверждение их выполнения предусмотрено средствами объективного технического контроля.

Метод анализа соответствия проектных параметров требованиям конструкторской и технологической документации. Данный метод основан на проверке релевантности параметров (38), выявленных в результате параметризации функционирования механического устройства раскрытия, с требованиями конструкторской и технологической документации в состоянии «как она есть».

Проверка релевантности параметров требованиям конструкторской документации проводится путем подтверждения того, что значения каждого из параметров при расчетах теоретической надежности выбраны в соответствие с нормативно-технической документацией, а в конструкторской документации предусмотрены достаточные требования для их подтверждения. Например, если используется конструкционный материал или конструктивное соединение с ненормированными характеристиками прочности, то в конструкторской документации должны быть указаны нормы прочности, включающие требования к назначению коэффициентов безопасности, экспериментальной отработке прочности, контролю и подтверждению прочности на этапах экспериментальной отработки, летной и штатной эксплуатации.

Для каждого параметра, обеспечивающего работоспособность и надежность, в конструкторской документации должны быть установлены требования для их достижения, подтверждения и контроля. Анализ проводят путем сличения контрольного списка параметров, на основании которых были сделаны расчеты надежности (38), с требованиями, которые установлены в конструкторской документации согласно видам документов по ГОСТ 2.102-2013 (чертежах деталей, сборочных чертежах, спецификациях чертежей, технических условиях, расчетах, программах и методиках и т.п.).

$$
-879-
$$


Проверка релевантности параметров требованиям технологической документации проводится путем сверки с требованиями конструкторской документации. Каждое из требований конструкторской документации должно быть без искажений и пропусков отражено в технологической документации. Особое внимание следует уделять случаям общей отсылки в чертежах к типовым технологическим процессам, когда там предусмотрены требования по детализации выполнения технологических операций, которые должны быть в обязательном порядке отражены в чертежах. В противном случае технолог может на свое усмотрение трактовать операции типового технологического процесса в технологической документации, полагая, что если конструктор не задал какое-либо из требований, то оно и неважно, тем самым может быть невольно нарушен конструкторский замысел, что способно привести к непредумышленному созданию условий для потенциальных отказов.

По результатам анализа конструкторской и технологической документации производят оценку рисков возникновения отказов вследствие неустановления требований, что может привести к недопустимым производственным дефектам при изготовлении или к отсутствию контроля (ненадлежащему контролю) параметров критичных элементов.

Метод оценки рисков возникновения отказов вследствие неустановления требований в конструкторской и технологической документации. Любое неустановленное требование в конструкторской или технологической документации должно быть оценено исходя из доминирующего вклада в суммарный риск возникновения отказов. Ранжирование рисков производится исходя из оценки значимости вклада того или иного потенциального события в совокупный риск отказов, связанных с неустановлением соответствующих требований. Для количественной оценки значимости рисков могут быть использованы методы, изложенные в ГОСТ Р ИСО 11231-2013 (метод ранжирования рисков) или ГОСТ 27.310-95 (метод балльных оценок критичности отказов).

Практика проведения анализов надежности показывает, что оценка рисков возникновения отказов вследствие неустановления требований в конструкторской и технологической документации является лишь одной из проблем анализа надежности [2]. В действительности, как правило, не проводят параметризацию функционирования механических устройств раскрытия, как следствие, не всегда выполняются расчеты параметров, необходимые для определения полной теоретической надежности (38). В этой связи непроведение расчетов параметров оценивается как неустановление требований в конструкторской документации, что должно быть расценено как невыполнение, ненадлежащее выполнение или отсутствие документального подтверждения свойств критичных элементов, влияющих на надежность. При этом проявление или непроявление заданных свойств критичных элементов рассматривается как событие. Вероятность совершения каждого из таких событий $P\left(C_{i}\right)$ определяется формулой

$$
P\left(C_{i}\right)=\alpha_{i} R_{i}
$$

где $\alpha_{i}$ - корректирующий коэффициент, определяемый экспертным путем в зависимости от результатов расчетов теоретической надежности и анализа соответствия проектных параметров требованиям конструкторской и технологической документации; $R_{i}$ - расчетная величина вероятности безотказной работы для каждого $i$-го вида отказа в формуле (38).

$$
-880-
$$


На практике для определения корректирующих коэффициентов $\alpha_{i}$ за основу может быть взят метод балльных оценок критичности отказов по ГОСТ 27.310-95, устанавливающий взаимосвязь между видами отказов по вероятности их возникновения и значениям ожидаемой вероятности:

$$
\alpha_{i}=1-Q_{i}
$$

где $Q_{i}$ - ожидаемая вероятность отказа $i$-го элемента согласно шкале балльных оценок критичности отказов по ГОСТ 27.310-95 (Приложение В).

Для вычисления ВБР с учетом рисков возникновения отказов (39) следует применять формулу

$$
P(\mathrm{C})=\prod_{i=1}^{n} P\left(C_{i}\right) .
$$

Если требования конструкторской и технологической документации, необходимые для проявления $i$-х свойств, определены в полном объеме, то в формуле (35) значения надежности по соответствующему параметру функционирования принимают равными расчетноэкспериментальным значениям. В этом случае считается, что риск отказа из-за отсутствия требований в документации ничтожно мал, тогда $Q_{i} \approx 0$, соответственно, $\alpha_{i} \approx 1$ и в формуле (41) $P\left(C_{i}\right)=R_{i}$ (этот случай дополняет ГОСТ 27.310-95). Если условия, необходимые для проявления $i$-х свойств, определены не в полном объеме, то в формуле (35) значения надежности по соответствующему параметру функционирования принимают равными расчетноэкспериментальным значениям, а окончательная оценка надежности производится по формуле (41) с учетом (40). Если вычисления показателей $R_{i}$ по какой-либо причине не проведены, то их значения в формуле (40) принимают условно равными $R_{i} \approx 1$, а риски отказов в формуле (41) из-за необоснованности параметров (показателей) учитывают при выборе корректирующих коэффициентов $\alpha_{i}$.

Метод оценки надежности с учетом влияния на надежность мероприятий по предупреждению отказов. Если в результате проведения анализа и оценки надежности по формулам (35) или (41) расчетное значение показателя безотказности окажется ниже требуемого значения, необходимо пересмотреть конструкторско-технологические требования надежности с корректировкой границ диапазонов значений показателей или параметров, поменять структурную модель надежности, изменить принятые конструктивные и технологические решения, выполнить недостающие расчеты и т.п. После этого следует провести итерационный анализ надежности с оценкой расчетного значения безотказности вплоть до выполнения заданного условия надежности.

Результаты апробирования технологии. Представленная методика анализа надежности апробирована при разработке механизмов одноразового срабатывания различных типов как для КА, так и для механизмов гидроавтоматики однократного срабатывания технологического оборудования нефтяных скважин [2].

В результате применения представленного анализа были выявлены конструкторские и технологические ошибки в технической документации; произведена оценка эффективности расчетно-экспериментальной отработки конструкции изделия; определена достаточность

$$
\text { - } 881-
$$


установленных требований в конструкторской документации; выявлены недопустимые сочетания параметров конструкции из-за конструктивных ограничений, условий изготовления и контроля; сделаны выводы об отказоспособности изделий; произведена оценка выполнения заданных требований к надежности; выданы рекомендации по изменению конструкции для обеспечения заданной надежности изделий. Полученные результаты позволяют своевременно принимать обоснованные конструкторские решения при проектировании изделий в режиме on-line с учетом их заданной надежности. Это способно сократить издержки на доработку изделий по результатам отработочных испытаний и снизить число отказов, в т.ч. из-за редких причин возникновения.

Заключение. Приведенная технология проектирования с учетом надежности, основанная на параметризации и проведении конструкторско-технологического анализа надежности, позволяет достигать безотказность механических устройств раскрытия whp $R \equiv 1$ вне зависимости от типа серийного производства. При этом потенциальные отказы могут быть с успехом устранены или смягчены на самых ранних стадиях жизненного цикла (проектировании и конструировании), а возможные риски возникновения отказов - своевременно оценены.

Учитывая, что с помощью рассмотренной обобщенной параметрической модели функционирования может быть параметризировано практически любое изделие, а в основу методики КТАН заложен функциональный подход (абстрагированный от конкретного конструктивного исполнения изделия), представленная технология проектирования может быть использована при разработке высоконадежных систем различного назначения, например механизмов многоразового срабатывания, электромеханических устройств, электронных сборок, силовых и прецизионных конструкций и т.п. В первую очередь, это относится к изделиям, имеющим заданную безотказность выше 0,999, которую сложно обеспечить с помощью обычных технологий проектирования и невозможно подтвердить в условиях единичного производства.

\section{Список литературы}

[1] Space Vehicle Mechanisms - Elements of Successful Design. Edited by Peter L. Conley. NJ: John Wiley \& Sons, 1998. 794 p.

[2] Похабов Ю.П. Теория и практика обеспечения надежности механических устройств одноразового срабатывания. Красноярск: Сиб. федер. ун-т, 2018. 338 с. [Pokhabov Yu.P. Theory and practice of ensuring the reliability of mechanical devices one-time operation, Krasnoyarsk, 2018, 338 p. (in Russian)]

[3] Кузнецов А.А., Золотов А.А., Комягин В.А. и др. Надежность механических частей конструкции летательных аппаратов. М.: Машиностроение, 1979. 144 с. [Kuznetsov A.A., Zolotov A.A., Komyagin V.A. et al. Reliability of mechanical parts of the aircraft structure, Moscow, 1979, 144 p. (in Russian)]

[4] Космические вехи / под ред. Н.А. Тестоедова. Красноярск: ИП Суховольская Ю.П., 2009. 704 c. [Space milestones / Edited by N.A. Testoyedov, Krasnoyarsk, 2009, 704 p. (in Russian)]

[5] Болотин В.В. Прогнозирование ресурса машин и конструкиий. М.: Машиностроение, 1984. 312 c. [Bolotin V.V. Prognozirovaniye resursa mashin i konstruktsiy, Moscow, Mashinostroyeniye, 1984, 312 p. (in Russian)] 
[6] Севастьянов Н.Н., Андреев А.И. Основы управления надежностью космических аппаратов с длительными сроками эксплуатации. Томск: Издат. Дом ТГУ, 2015. 266 с. [Sevast'yanov N.N., Andreyev A.I. Fundamentals of reliability management of spacecraft with long service life, Tomsk, 2015, 266 p. (in Russian)] 